\title{
GPPS-CH-2020-0094
}

\section{AEROACOUSTIC NUMERICAL INVESTIGATIONS OF A SCALED COMPRESSOR CASCADE}

\author{
Stefanie Lohse ${ }^{1}$ \\ Leibniz Universität Hannover \\ stefanie.lohse@stud.uni-hannover.de \\ Hannover, 30167, Germany
}

\author{
Régis Koch \\ Mechanical Engineering \\ Université de Sherbrooke \\ regis.koch@usherbrooke.ca \\ Sherbrooke, J1K 2R1, QC, Canada
}

\author{
Stéphane Moreau \\ Mechanical Engineering \\ Université de Sherbrooke \\ stephane.moreau@usherbrooke.ca \\ Sherbrooke, J1K 2R1, QC, Canada
}

\author{
Felix Fischer ${ }^{2}$ \\ Institute of Turbomachinery \\ and Fluid Dynamics \\ Leibniz Universität Hannover \\ felix.fischer@tfd.uni-hannover.de \\ Garbsen, 30823, Germany
}

\author{
Joerg R. Seume \\ Institute of Turbomachinery \\ and Fluid Dynamics \\ Leibniz Universität Hannover \\ seume@tfd.uni-hannover.de \\ Garbsen, 30823, Germany
}

\section{ABSTRACT}

In order to further develop technologies to reduce noise emissions of aero engines, an understanding of the noise propagation through compressor blade rows of modern turbofan engines is of major importance. To enable more detailed experimental investigations of the sound propagation in aero engines, engine components or stages have to be scaled for an installation into test rigs that allow for experiments under acoustically optimized boundary conditions. The main focus of the present work is thus to discuss a scaling approach that ensures both aerodynamic and aeroacoustic similarity between a given test rig and engine. For that purpose, a stator row of a four-stage high-speed axial compressor (4AC) based on the test rig at the Institute of Turbomachinery and Fluid Dynamics (TFD) at the Leibniz University Hanover is scaled to fit into the TFD's Aeroacoustic Wind Tunnel (AWT). Numerical investigations based on multiple modeling approaches are performed to verify a similar aeroacoustic behaviour in both test rigs. Reynolds-Averaged Navier-Stokes (RANS) and Unsteady-RANS simulations are carried out to assess the aerodynamic characteristics of the blade rows. The aeroacoustic modelling consists of simulations with an Euler acoustic solver to compare the modal transmis-

\footnotetext{
${ }^{1}$ The presented work was divided equally between the Institute of Turbomachinery and Fluid Dynamics in Hanover and the Mechanical Engineering Department in Sherbrooke (stefanie.lohse@usherbrooke.ca).

${ }^{2}$ Address all correspondence to this author.
}

sion behaviour through the blade rows. The CFD results show that aerodynamic similarity was achieved. Moreover, the CAA results indicate that the acoustic transmission behaviour of the blade row could be matched for both test rigs.

\section{INTRODUCTION}

To continuously improve and extend existing sound transmission models, experimental investigations of sound propagation in turbo engines are of utmost importance. However, most turbomachinery test rigs are designed with a focus on aerodynamic variability rather than aeroacoustically optimized boundary conditions such as low aeroacoustic reflections within the test rig. If local effects such as sound propagation through a single blade row are of interest for the global machine performance, a multi stage test rig is often not adequate for accurate acoustic measurements.

Such a case was reported by Hellmich and Seume (Hellmich and Seume, 2008) when they first described the phenomenon of acoustic resonance inside the TFD's 4AC test rig. They could show that the resonance was a result of a standing acoustic wave that formed in between the rotating blade rows of the compressor. Although their modeling approach is in good agreement with their experimental findings regarding the reflections at the rotor rows, the transmission behaviour of the stator rows was underestimated. In order to get a better understanding of the acoustic transmission behaviour of the stator 
blades in the $4 \mathrm{AC}$, future acoustic measurements at the $4 \mathrm{AC}$ are accompanied by isolated measurements of the stator's acoustic transmission behaviour in the TFD's AWT. As the AWT's and 4AC's duct diameters do not coincide, a scaling approach is required to ensure comparable experimental results in both test rigs. In the present paper, such a scaling approach is derived from the most common aeroacoustic modeling approaches in aero engines. With the performed numerical simulations it is not only intended to investigate the aerodynamic behaviour of a stator row implemented in both test rigs but also to compare the acoustic transmission behaviour and to show the aeroacoustic similarity.

Previously, Hurfar et al. (Hurfar et al., 2015) have studied the aeroacoustic effects of scaling a symmetric hard-walled annular duct assuming a constant cross-section and a uniform axial flow. Notable work on the velocities and corresponding angles of modal sound propagation is presented with the conclusion that a similar propagation can be achieved by considering the hub-to-tip ratio as well as Mach and Helmholtz number as dimensionless parameters. The mathematical modeling of sound transmission through blade rows is described by Smith (Smith, 1972). The model approaches a two-dimensional blade row with a cascade of thin, flat plates and analyzes the amount of transmitted and reflected sound of an impinging acoustic wave.

\section{FUNDAMENTALS}

\section{Sound Propagation in Ducts}

The modeling approach commonly applied to describe the sound propagation of acoustic modes in an axisymmetric duct is based on the solution of the Pridmore-Brown equation:

$$
\hat{p}=\sum_{m=-\infty}^{\infty} \sum_{n=0}^{\infty}\left(A_{m n}^{+} e^{i k_{z, m n}^{+} z}+A_{m n}^{-} e^{i k_{z, m n}^{-} z}\right) \cdot f_{m n}(r) \cdot e^{i m \varphi} \cdot e^{-i \omega t}
$$

with the azimuthal mode order $m$, the radial mode order $n$, the complex amplitudes $A_{m n}^{ \pm}$and axial wave numbers $k_{z, m n}^{ \pm}$ wherein the superscript indicates whether the respective mode propagates upstream (-) or downstream $(+)$. The radial eigenfunction $f_{m n}(r)$ is given as:

$$
f_{m n}(r)=J_{m}\left(s_{m n} \frac{r}{R_{S h r}}\right)+Q_{m n} \cdot Y_{m}\left(s_{m n} \frac{r}{R_{S h r}}\right)
$$

The radial eigenfunction consists of the Bessel functions of first $\left(J_{m}\right)$ and second $\left(Y_{m}\right)$ kind with the respective order $m$. The radial eigenvalues $s_{m n}$ and $Q_{m n}$ depend on the mode orders $m$ and $n$ and the hub-to-tip ratio $\sigma=\frac{R_{H u b}}{R_{S h r}}$.

Under the assumption of a uniform axial flow and a rigid body swirl distribution for the circumferential flow component, the axial wave numbers can be expressed as:

$$
\begin{aligned}
k_{z, m n}^{ \pm} & =\frac{-M a_{z} \cdot\left(k-\frac{m \cdot M a_{\varphi, S h r}}{R_{S h r}}\right)}{1-M a_{z}^{2}} \\
& \pm \frac{\sqrt{\left(k-\frac{m \cdot M a_{\varphi, S h r}}{R_{S h r}}\right)^{2}-\left(1-M a_{z}^{2}\right) \cdot\left(\frac{s_{m n}}{R_{S h r}}\right)^{2}}}{1-M a_{z}^{2}}
\end{aligned}
$$

\section{Sound Transmission Model by Smith}

Several approaches for the analytical modelling of the reflection and transmission behaviour through blade rows have been developed since the 1970s. In the present paper, the model by Smith (Smith, 1972) is applied which analyzes a two-dimensional blade row for any type of incoming gust and applies a distribution of bounded vortices along the vane chord. Then, the concept of cascade waves, which facilitates the computation of the transmission and reflection coefficients, is introduced and methods to predict the forces on the blades are developed. Smith's approach gives a solution for both the scattered acoustic field upstream or downstream of the cascade with a steady or unsteady loading and the vortical field induced by flutter and bending excitation or incident vortical gusts and acoustic waves.

The model by Smith is an abstraction which simplifies a stator row as an unwrapped, infinite 2D cascade of flat plates with zero thickness and the pitch $s$ between adjacent vanes, chord length $c$ and stagger angle $\alpha_{s}$ as depicted in Fig. 1 .

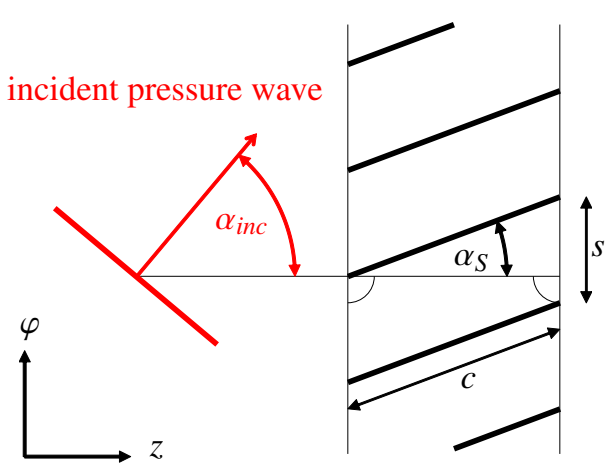

\section{Figure 1 Generic blade row for the representation of the relevant input parameters of acoustic 2D transmission modeling with plate models}

It is assumed that the flow is parallel to the plates. If an incoming acoustic wave (index inc) impinges the cascade at an angle $\alpha_{i n c}$, the wave is rather reflected (index $r$ ) or transmitted (index $t$ ). The transmission coefficient $T$ and reflection coefficient $R$ are then calculated using the complex amplitudes of the 
incoming, transmitted and reflected acoustic waves:

$$
T=\frac{\left|\bar{p}_{t}\right|}{\left|\bar{p}_{\text {inc }}\right|} \quad \text { and } \quad R=\frac{\left|\bar{p}_{r}\right|}{\left|\bar{p}_{\text {inc }}\right|}
$$

\section{SCALING APPROACH}

As the AWT's current design does not support rotating stages, a stator row from the $4 \mathrm{AC}$ is selected for geometric scaling. According to Hurfar et al. (Hurfar et al., 2015), the hub-to-tip ratio is of overall importance for the aeroacoustic similarity of the acoustic modes' radial sound pressure distribution. Therefore, the hub-to-tip ratios of the 4AC stator rows were compared with the fixed ratio of 0.66 in the AWT and the third stator row with a ratio of 0.7029 was finally chosen. Furthermore, it was advantageous that numerical results for the third stage of the axial compressor were available, which were used to extract the boundary conditions for numerical simulations. Table 1 summarizes the design parameters for the existing compressor blade. A more detailed description of the underlying geometry of the third stator of the $4 \mathrm{AC}$ can be found in (Hellmich and Seume, 2008) (Hellmich, 2008).

\section{Table 1 Design parameters for compressor blade}

\begin{tabular}{ll} 
blade number & 34 \\
incident flow angle & $36.04^{\circ}$ \\
stagger angle (at midspan) & $69.548^{\circ}$ \\
aspect ratio & 1.542 \\
solidity (at midspan) & 1.303 \\
hub-to-tip ratio & 0.7029 \\
\hline
\end{tabular}

To further approximate the conditions for an AWT setup, the hub radius of the actual stator blade was set to a uniform mean value, as depicted in Fig. 2. For the geometric scaling, the ratio between the shroud radius of the AWT and $4 \mathrm{AC}$ was used as a scaling factor: $R_{s h r, A W T} / R_{S h r, 4 A C}=1.47$. For this purpose, the center of mass of the $4 \mathrm{AC}$ blade was determined and the scaling factor was uniformly applied in all directions to obtain the geometry of the scaled blade; the number of blades was reduced also to 29

\section{Dimensional Analysis}

In order to achieve identical transmission and reflection behaviour based on a similar flow in both configurations for the 4AC and AWT, the studies by Smith (Smith, 1972) and Hurfar et al. (Hurfar et al., 2015) were consulted to select the parameters that were considered for the dimensional analysis.

The dimensional analysis was carried out to identify the characteristic numbers that define the problem at hand and
SHROUD

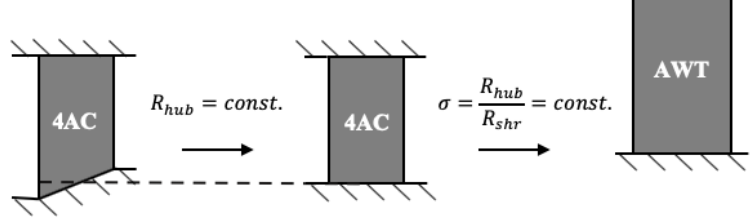

HUB

Figure 2 Process to design the scaled compressor blade.

must be kept constant in both configurations to achieve the same aerodynamic and aeroacoustic results after scaling. The analysis procedure was based on Buckingham's investigations (Buckingham, 1914). The present turbomachinery problem was described by eight independent variables with the corresponding units categorized in Tab. 2.

Table 2 List of variables with corresponding units

\begin{tabular}{lllc}
\hline & shroud radius $R_{\text {shr }}$ & $\mathrm{m}$ & $\mathrm{L}$ \\
geometric & blade height $h$ & $\mathrm{~m}$ & $\mathrm{~L}$ \\
variables & blade chord length $c$ & $\mathrm{~m}$ & $\mathrm{~L}$ \\
& pitch at midspan $s$ & $\mathrm{~m}$ & $L$ \\
\hline kinematic and & stream velocity $v$ & $\mathrm{~m} / \mathrm{s}$ & $\mathrm{L} \mathrm{T}^{-1}$ \\
dynamic variables & frequency $f$ & $1 / \mathrm{s}$ & $\mathrm{T}^{-1}$ \\
\hline \multirow{2}{*}{ fluid variables } & kinematic viscosity $v$ & $\mathrm{~m}^{2} / \mathrm{s}$ & $\mathrm{L}^{2} \mathrm{~T}^{-1}$ \\
& speed of sound $a_{0}$ & $\mathrm{~m} / \mathrm{s}$ & $\mathrm{L} \mathrm{T} \mathrm{T}^{-1}$ \\
\hline
\end{tabular}

As the pressure and temperature depend on the density and speed of sound, these two variables were not listed additionally. All relevant variables were expressed in terms of the two reference dimensions: length (L) and time (T).

Thus, there were six degrees of freedom to derive six characteristic numbers according to the pi theorem by Buckingham (Buckingham, 1914). The following non-dimensional numbers were obtained: aspect ratio $\frac{h}{c}$, blade solidity $\sigma_{s}=\frac{c}{s}$, Reynolds number $\operatorname{Re}=\frac{\rho v c}{\eta}$, Mach number $M a=\frac{v}{a_{0}}$, Helmholtz number $H e=\frac{2 \pi \cdot f R_{s h r}}{a_{0}}$ and Strouhal number $S r=\frac{f c}{v}$.

Beside the geometric ratios, which match for both stator blades, it has been shown that an aerodynamic and aeroacoustic similarity can be achieved if the Reynolds number, Mach number, Helmholtz number and Strouhal number were kept equal in both configurations. This means that two transient flows of frictional compressible fluids around geometrically similar contours are similar if their ratios of (i) inertial forces 
to viscous forces, (ii) flow velocity to local speed of sound, (iii) characteristic length to wavelength and (iv) inertial forces due to the local acceleration of the flow to inertial forces due to the convective acceleration are equal. Instead of the Strouhal number, the more commonly used hub-to-tip ratio $\sigma$ was considered as a resemblance parameter in accordance with Hurfar et al. (Hurfar et al., 2015). This is possible as the Strouhal number can be converted into the hub-to-tip ratio using the remaining dimensionless parameters (detailed conversion in the appendix). These six dimensionless quantities can be found again in the modelling describing sound propagation in circular/annular ducts and through blade rows. Using the definition of the free-field wave number $k=\frac{\omega}{a^{0}}$ and the Helmholtz number, Eq. 3 can be rewritten as

$$
\begin{aligned}
k_{z, m n}^{ \pm} & =\frac{-M a_{z} \cdot\left(H e-m \cdot M a_{\varphi, S h r}\right)}{R_{S h r} \cdot\left(1-M a_{z}^{2}\right)} \\
& \pm \frac{\sqrt{\left(H e-m \cdot M a_{\varphi, S h r}\right)^{2}-\left(1-M a_{z}^{2}\right) \cdot s_{m n}^{2}}}{R_{S h r} \cdot\left(1-M a_{z}^{2}\right)} .
\end{aligned}
$$

As the radial eigenvalue $s_{m n}$ only depends on the respective mode orders and the hub-to-tip ratio, the axial wave number scales inversely proportional to the shroud radius under the assumption that all dimensionless quantities are held constant. Using the definition of the acoustic propagation angle in accordance with Behn et al. (Behn et al., 2016).

$$
\cos \left(\alpha_{i n c}^{ \pm}\right)=\frac{k_{z, m n}^{ \pm}}{k-M a_{z} \cdot k_{z, m n}^{ \pm}}=f\left(H e, M a_{z}, M a_{\varphi}, m, n, \sigma\right)
$$

it can be shown that the propagation angle of a given mode $[m, n]$ is equal for two different ducts as long as the dimensionless quantities $\mathrm{He}, M a_{z}, M a_{\varphi}$ and $\sigma$ were kept constant. This propagation angle is of importance in regard to the transmission and reflection behaviour of acoustic modes propagating through a blade row as modelled by Smith. Beside this, the aspect ratio, the plate's staggering angle, the Mach number of the plate-parallel flow and the frequency parameter that equals the blade's Strouhal number are required input parameters for Smith's transmission model (Smith, 1972).

Based on the similarity of these six dimensionless parameters, the existing 4AC's aerodynamic operating point has been scaled at midspan. A non linear equation system was devised and solved with the iterative method of least-square's error to obtain the scaled temperature and pressure and a conservative estimate of the scaling of flow-field parameters between the $4 \mathrm{AC}$ and AWT configuration was drawn. The frequency was scaled based on the Helmholtz number and the flow-field parameters were further varied to set up the boundary condition for the performed numerical simulations.

\section{METHODOLOGY Numerical Model}

A fully three-dimensional compressible steady-state RANS simulation was performed to calculate the resulting flow-field. The inflow Mach number equals 0.5 and the Reynolds number based on chord equals approximately $3.7 \times 10^{5}$. The numerical setup was derived from the actual compressor, which led to a domain geometry with a short distance upstream between the inlet and leading edge. The inlet was located at a distance of $0.24 c$ upstream of the leading edge and the domain outlet at $1.4 c$ downstream of the trailing edge. A single-passage domain was simulated with a given periodicity. For the scaled blade, the same axial distances were used for the numerical setup to force a similar boundary layer growth in the axial direction. With the RANS simulations transient phenomena were observed as a result of flow separation and a subsequent URANS simulation was performed to improve the convergence behaviour. For the unsteady simulations, the RANS results were used as an initial solution and an implicit method was used to simulate the time-dependent flow-field in both configurations.

\section{Grid Topology}

For both blade configurations, a structured mesh was generated for the steady and unsteady simulations. The two meshes consist of approximately $1.13 \times 10^{6}$ cells and a near wall cell size that provides a $y^{+}$value smaller than one. Another significant parameter for the mesh generation is the cell width $w$. According to Weckmüller et al. (Weckmüller et al., 2009), a numerical scheme with a second order spatial scheme requires a spatial discretization resolution of 40 to 50 cells per wavelength to minimize the numerical damping effects when calculating the acoustic propagation. To develop a resource-saving model without unnecessary cells, a mesh with 40 cells per wavelength for all cut-on modes within the excited frequency in the domain was chosen. Furthermore, a grid convergence study was carried out, which examines a coarsened and refined mesh as described in detail in a following subsection.

\section{Simulation Settings}

The steady and unsteady simulations were performed using the software ANSYS CFX 19.2. Regarding the solver settings, an advection scheme of second order was chosen for the numerical discretization. To limit the convergence of the numerical solution, a physical time step was fixed to $10^{-3}$ s for the RANS calculations. For the unsteady simulations, a time step of $10^{-4} \mathrm{~s}$ was chosen based on the stator pitch and the exit relative velocity of the upstream rotor in the actual compressor. The number of sub-iterations was set to 10 and the simulation duration included two full rotations of the upstream rotor. Moreover, the turbulence model $k-\omega$-SST was used to model the turbulence phenomena and the turbulence intensity at the inlet was set to a medium level of 5\%. In addition, the Kato-Launder modification was applied to eliminate the unphysical production of turbulence in stagnation points. To model the transition 
especially near walls, the $\gamma-R e_{\theta}$ model was selected in both configurations.

The boundary conditions at the inlet were defined by a total pressure as well as total temperature distribution depending on the radius. Furthermore, the flow direction was specified by normalized velocity profiles in cylindrical coordinates. At the outlet a specified mass flow rate was imposed. The walls and blade surface were modeled as adiabatic and with a no-slip condition. The radial distributions of flow-field parameters, which were given as boundary conditions for the simulations with the 4AC blade, were extracted from the previous numerical simulation. Moreover, results of the investigation on acoustic resonance in the 4AC by Hellmich and Seume (Hellmich and Seume, 2008) have been used as an orientation for the numerical setup. The same throttled operating point was modelled and adjusted for the AWT blade using the assumptions made with the scaling approach to verify a similar aerodynamic behaviour. The specified mass flow was increased and the total quantities were decreased to obtain the same Mach and Reynolds number in both configurations. The adaptions were reasonable regarding the area ratio greater than one between both cases due to the upscaling.

The Euler acoustic solver ACTRAN 18.1 developed by Free Field Technologies was used to model the sound propagation through the blade rows under consideration of a time invariant mean flow. For these simulations, the mean flow was extracted from the acquired RANS solutions and mapped onto the acoustic domain. The meshes used corresponded to those used in the (U)RANS simulations. The acoustic field was excited using an acoustic boundary condition at the domains inlet region using non-reflecting boundary conditions to minimize numerical errors. In addition, all walls such as the blade surface were modeled as sound-reflecting. The solution for the acoustic field was achieved in the frequency domain using the implemented MUMPS solver (Antwerpen et al., 2008).

\section{Grid Convergence Study}

In order to investigate the mesh independence of the acquired flow solutions, the grid convergence index (GCI) as defined by Roache (Roache, 1994) was calculated. For the integral flow variable of interest, the forces acting on the blade surfaces were chosen to evaluate the convergence criterion for the unsteady simulations as suggested by Moreau et al. (Moreau et al., 2005). The GCI is defined as:

$$
\mathrm{GCI}=\frac{F_{S} \cdot e_{a}}{u^{d}-1}
$$

Therein, $F_{S}$ is a safety factor to ensure a conservative approximation of the grid independence, $e_{a}$ is the relative change of the respective integral quantity in between two simulations with varying grids, $r$ is the relative change of the grid size (resolution) and $d$ is the observed order of accuracy.

The grid study included a coarsened and a refined mesh compared to the reference meshes of the $4 \mathrm{AC}$ and the AWT respectively. The directed scaling factor of $u_{r}=u_{\varphi}=u_{z} \approx 0.8$ was set to meet a global refinement factor for the main flow of $u \approx 0.5$ for all meshes. The wall normal length of the cell closest to the wall was not adjusted so as not to influence the $y^{+}$value and thus the numerical treatment of the boundary layers. Table 3 lists the most relevant intermediate results and the GCIs for the normal (y) and tangential (z) components of the blade forces for the used numerical 4AC and the AWT setups. Therein, the safety factor $F_{S}$ was assigned a value of 1.25 in accordance with the ASME V\&V Committee (Committee, 2009).

Table 3 Grid Convergence Index of the blade forces

\begin{tabular}{lllll}
\hline & $\boldsymbol{F}_{\boldsymbol{y}, \mathbf{4 A C}}$ & $\boldsymbol{F}_{\boldsymbol{z}, \mathbf{4 A C}}$ & $\boldsymbol{F}_{\boldsymbol{y}, \boldsymbol{A W T}}$ & $\boldsymbol{F}_{\boldsymbol{z}, \boldsymbol{A} W \boldsymbol{T}}$ \\
\hline $\boldsymbol{e}_{\boldsymbol{a}}^{\mathbf{2 1}}$ & 0.0067 & 0.0093 & 0.0073 & 0.0132 \\
$\boldsymbol{e}_{\boldsymbol{a}}^{\mathbf{3 2}}$ & 0.0018 & 0.0050 & 0.004 & 0.0071 \\
$\boldsymbol{d}$ & 1.4441 & 0.5124 & 0.6311 & 0.6612 \\
$\boldsymbol{e}_{\boldsymbol{e x t}}^{\mathbf{2 1}}$ & 0.0094 & 0.0260 & 0.0183 & 0.0313 \\
$\boldsymbol{e}_{\boldsymbol{e x t}}^{\mathbf{3 2}}$ & 0.0028 & 0.0169 & 0.0111 & 0.0186 \\
$\boldsymbol{G C I}^{\mathbf{2 1}}$ & -0.0119 & -0.0333 & -0.0234 & -0.0405 \\
$\boldsymbol{G C I}^{\mathbf{3 2}}$ & -0.0035 & -0.0215 & -0.0141 & -0.0237 \\
\hline
\end{tabular}

As shown in Tab. 3, the relative error between the forces estimated with an Richardson extrapolation and the forces taken from the simulations $\left(e_{e x t}^{32}\right)$ is $0.28 \%$ for the normal force and $1.69 \%$ for the tangential force of the 4AC simulations and $1.11 \%$ for the normal force and $1.86 \%$ for the tangential force of the AWT simulations.

\section{RESULTS AND DISCUSSION Overall Mean Flow and Performances}

To obtain a converged solution for the steady simulation, the domain length upstream of the blade (inflow) was varied. It was observed that the inflow length is of major importance as the boundary layer grows in axial direction. An increased inflow length resulted in a larger separation area on the blade suction side as well as higher fluctuations of the flow variables. In addition, a smearing of the inlet boundary conditions was visible when increasing the distance between inlet and blade leading edge. Satisfactory convergence has finally been achieved for an added incidence angle of -5 degree to the given circumferential velocity profile at the inlet. The residual was reduced to $8 \times 10^{-6}$ and settled fluctuations in the residual courses were achieved.

For the unsteady simulations the integrated forces on the blade surface were monitored as it was established by Moreau et al. (Moreau et al., 2005). A converged periodic behavior has been exhibited by polar curves when plotting the normal 
(y) and tangential (z) forces. Convergence was reached when the polar curves were overlapping and a deviation of less than $1 \%$ (estimated numerical error according to the grid study) was achieved.

As the flow imposed at the inlet is influenced by the wake of the upstream located rotor, it is not uniform but swirling. Figure 3 shows the areas on the blade suction side where the wall shear stress in axial direction $\tau_{\omega, z}$ is less or equal zero. A corner vortex triggers a flow separation in the hub region increasing in axial and spanwise direction. The separation was observed at the same location of the 4AC and AWT blade surface. Furthermore, it has to be kept in mind that the original $4 \mathrm{AC}$ blade was designed with a varying hub radius and is therefore not optimized for a constant hub geometry. As a result of the simulated operating point and in particular the given chord based Reynolds number, the dominant transition effects and associated flow separation were expected.

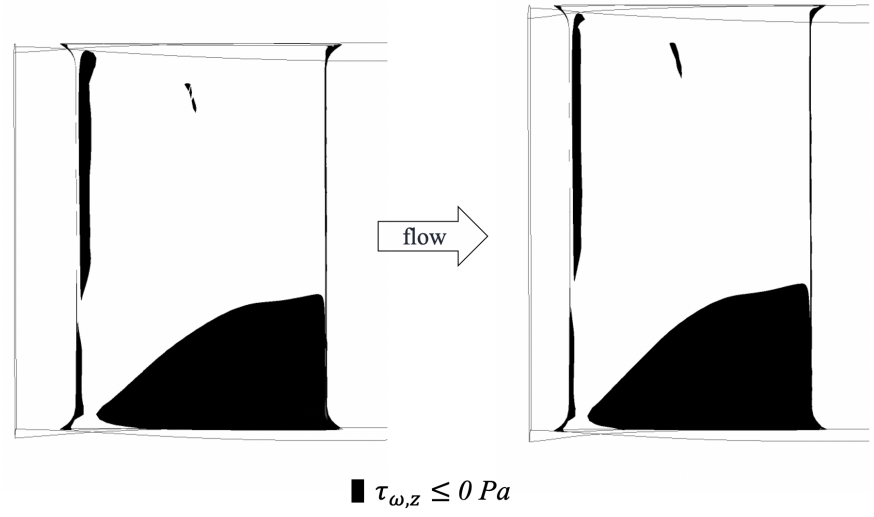

Figure 3 Areas of flow separation on the 4AC blade (left) and AWT blade (right) suction side (RANS simulations).

The flow-field was further investigated with a comparison of the Mach number distribution for $M a \leq 0.35$ at $25 \%$ blade height (Fig. 4). A separation bubble was depicted which has been induced by the occurring corner vortex. The separation bubble is characterized by a flow regime with significantly lower momentum as displayed by the low Mach numbers. Table 4 compares the overall performance of the cascade. It shows that the scaling yielded the same pressure ratio in the RANS and URANS simulations. The URANS results differ by $0.55 \%$ (AWT blade) respectively $0.28 \%$ (4AC blade) from the RANS.

To validate the aerodynamic similarity between the existing and scaled compressor blade, the Mach and Reynolds number were compared (Fig. 5, 6). Their radial distributions were obtained by extracting flow parameters in all simulations at the same axial position upstream of the leading edge and downstream of the trailing edge. An area average has been
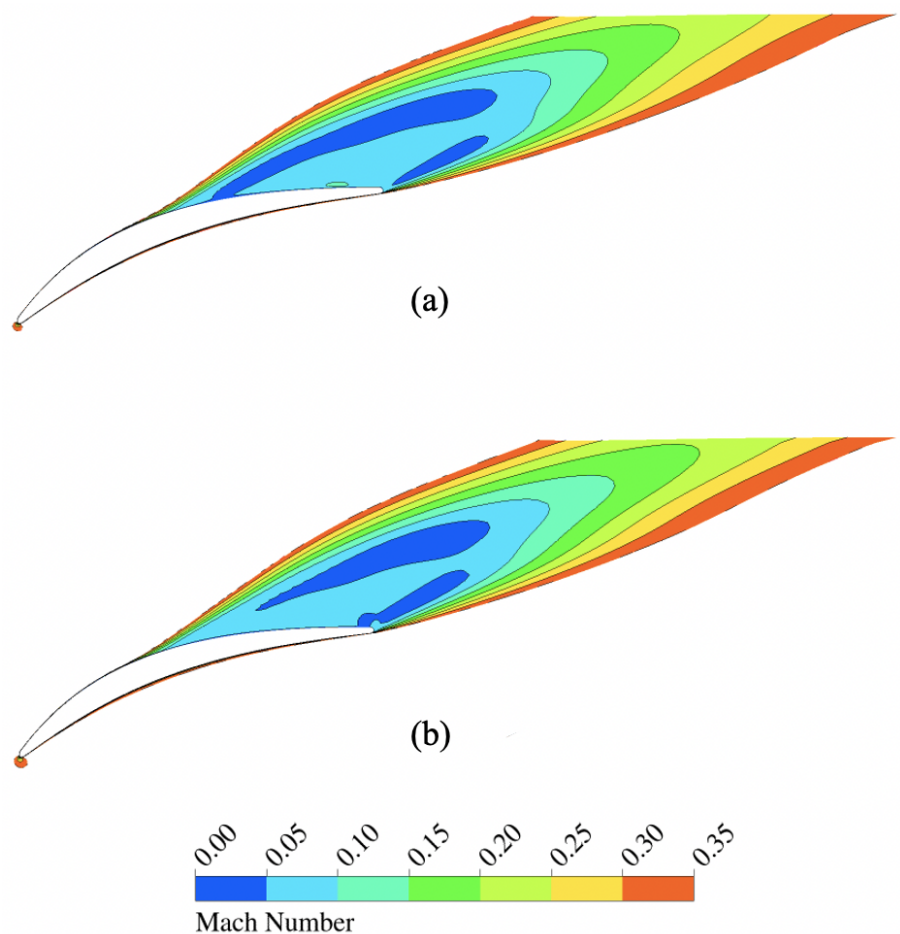

Figure 4 Comparison of the Mach number distribution ( $M a \leq 0.35)$ downstream of the blades at $25 \%$ span (RANS simulations) - (a): 4AC and (b): AWT.

Table 4 Comparison of the pressure ratio $\frac{p_{\text {out }}}{p_{\text {in }}}$

\begin{tabular}{lll} 
& RANS & URANS \\
\hline 4AC & 1.04016 & 1.03725 \\
AWT & 1.04049 & 1.03485 \\
\hline
\end{tabular}

applied in circumferential direction.

For both upstream Mach and Reynolds number profiles the RANS and URANS results in Fig. 5 (a) and Fig. 6 (a) coincide. The differences between their profiles in the range of $0-0.2$ $h_{\text {rel }}$ representing the characteristic numbers downstream in Fig. 5 (b) and Fig. 6 (b) are negligible. It is noticeable that the 4AC and AWT results upstream especially differ in the hub region. This can be explained with the obstruction of the flow channel as displayed in Fig. 4 that directly effects the flow upstream due to the limited axial dimension downstream.

With Fig. 7 the negative mean pressure coefficient $-c_{p}$ distribution around the blade is compared for all four simulations at three different spanwise positions (25\% span, $50 \%$ span and $75 \%$ span). Again it is shown that the RANS results predominantly correspond to the URANS results for the 4AC blade and AWT blade respectively. Any discrepancy between the steady-state and transient simulation results can be justified by the time dependency of flow parameters which is only respected in the URANS simulations. On the pressure side 


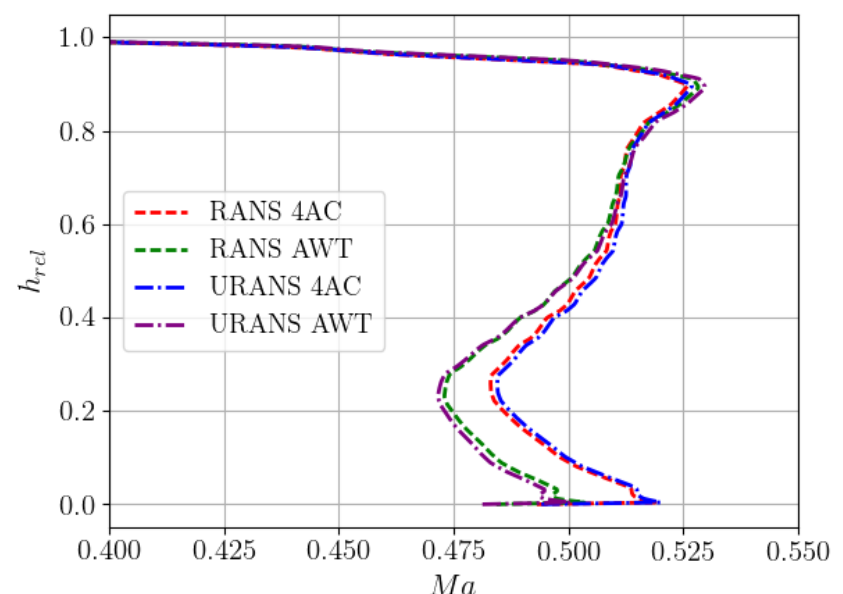

(a)

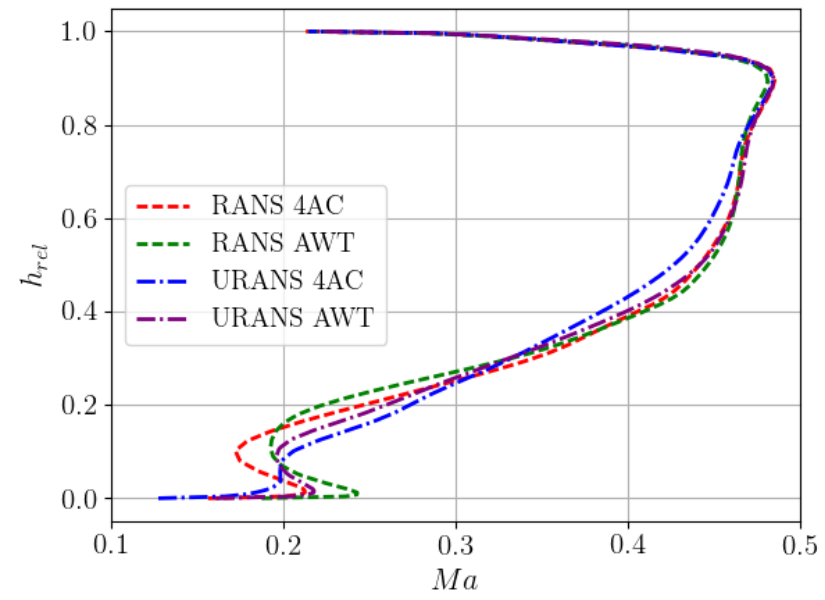

(b)

Figure 5 Comparison of the radial Mach number distribution at two axial positions - (a): upstream and (b): downstream of the blade.

the loading on the AWT blade deviates from the 4AC blade, however, the presented scaling approach has been validated with a comparison of simulation results obtained from several performed numerical simulations.

\section{Acoustic Transmission Properties}

To allow for a preliminary comparison of the acoustic transmission behaviour between the 4AC blade and the AWT blade, aeroacoustic simulations were conducted using ACTRAN 18.1. As the mean flow is only considered as a convective influence on the sound propagation but not as an acoustic source, the results permit an evaluation of the sound propagation through the blade row without consideration of the blades self-noise. For the scaling study at hand, simulations were carried out considering circumferential mode orders ranging from $m=-3$ to $m=3$ at a radial mode order of $n=0$ and a frequency of $1500 \mathrm{~Hz}(4 \mathrm{AC})$ and $945 \mathrm{~Hz}$ (AWT) respectively. The chosen $4 \mathrm{AC}$ frequency is of acoustic interest as this operation point

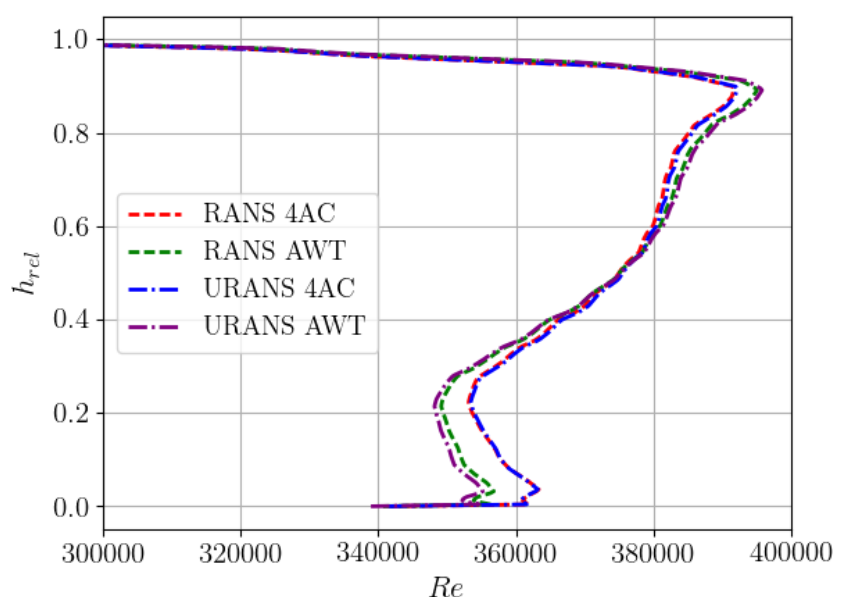

(a)

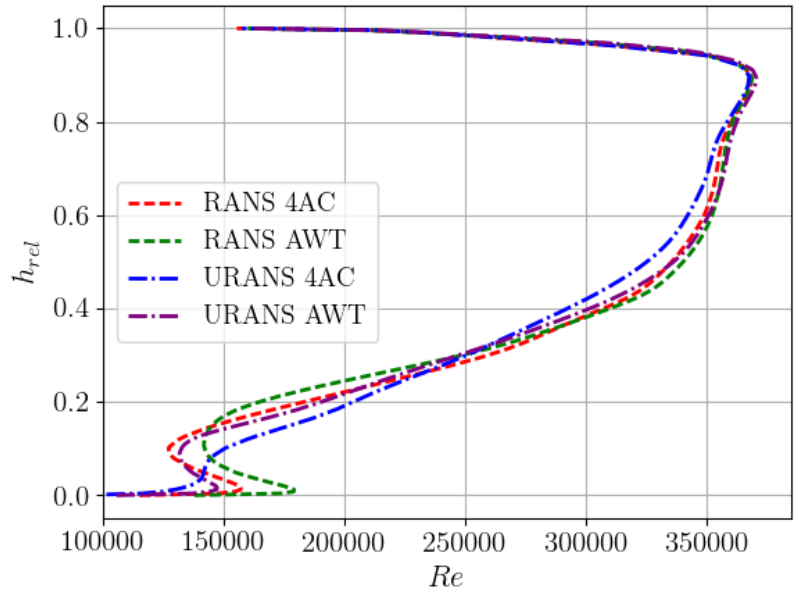

(b)

Figure 6 Comparison of the radial Reynolds number distribution at two axial positions - (a): upstream and (b): downstream of the blade.

has previously been studied by Hellmich and Seume (Hellmich and Seume, 2008). By keeping the Helmholtz number constant in both configurations, the scaled frequency was obtained. All propagable mode orders at these excitation frequencies were evaluated. Figure 8 shows the acoustic transmission coefficients of both blades as a function of the circumferential mode order of the incident wave. In accordance with Smith (Smith, 1972), the sound transmission coefficient $T$ is defined as the amplitude ratio of the transmitted and the incident wave as defined in Eq. 4.

These preliminary results show that a dominant part of the incoming wave propagates through the blade row in all examined cases, which is in good agreement with the experimental results of Hellmich and Seume (Hellmich and Seume, 2008). As for the comparison of the transmission behaviour, both blades show a fairly similar acoustic transmission behaviour in regard to the incident waves' circumferential mode order. The numerical values of the determined transmission coefficients 


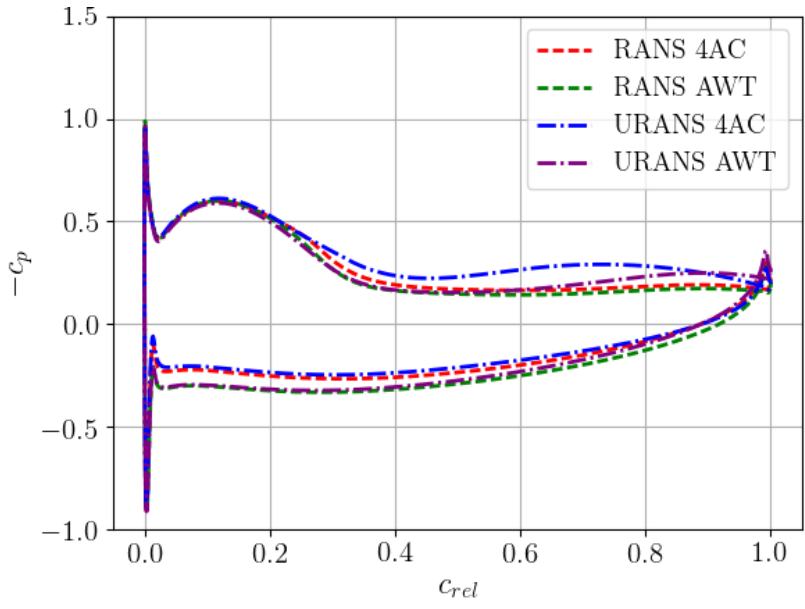

(a)

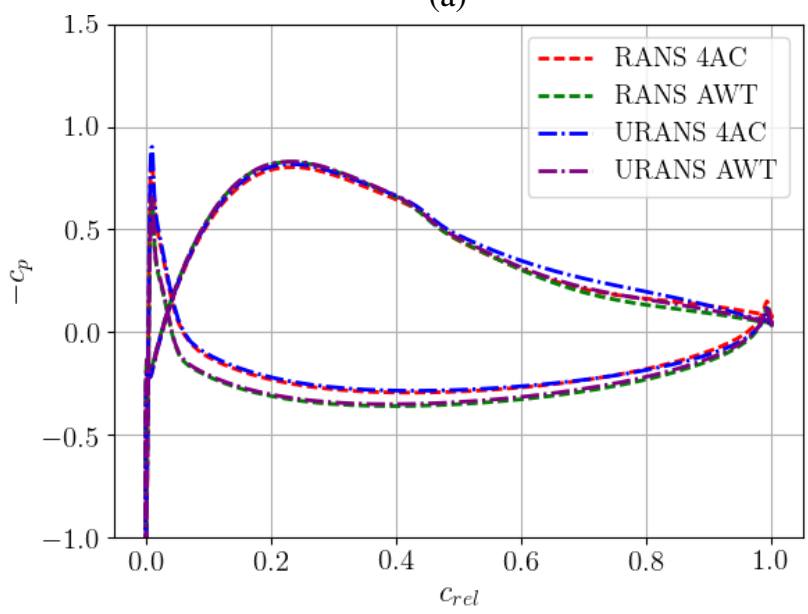

(b)

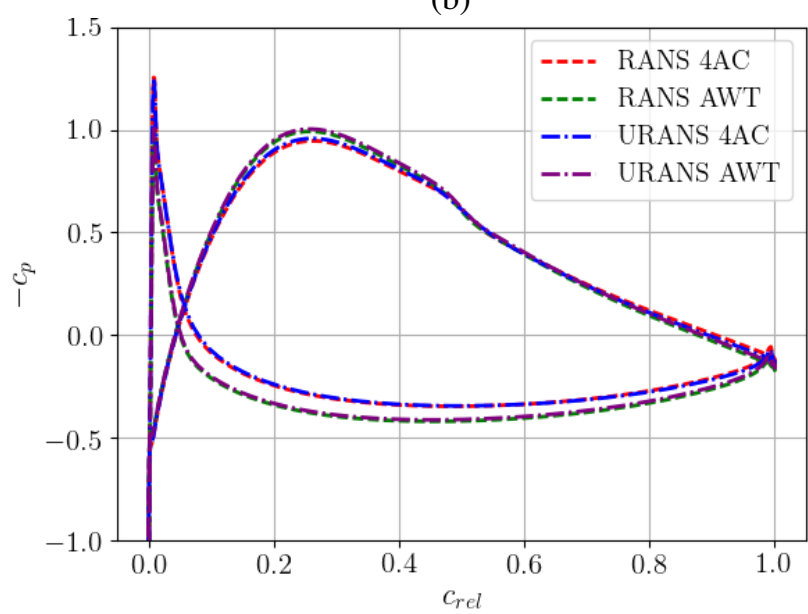

(c)

Figure 7 Comparison of the $C_{p}$ distribution at three spanwise positions - (a): hub (25\% span); (b): midspan (50\% span) and (c): shroud ( $75 \%$ span).

show a maximum deviation of $0.41 \%$ for the circumferential mode oder $m=3$ and a minimal deviation of $0.13 \%$ for the mode order $m=-1$. This can be explained with the differences in the hub-to-tip ratio, which has been identified as a relevant parameter for the acoustics, of approximately 0.04 between the $4 \mathrm{AV}$ and AWT and therefore led to insignificantly different results.

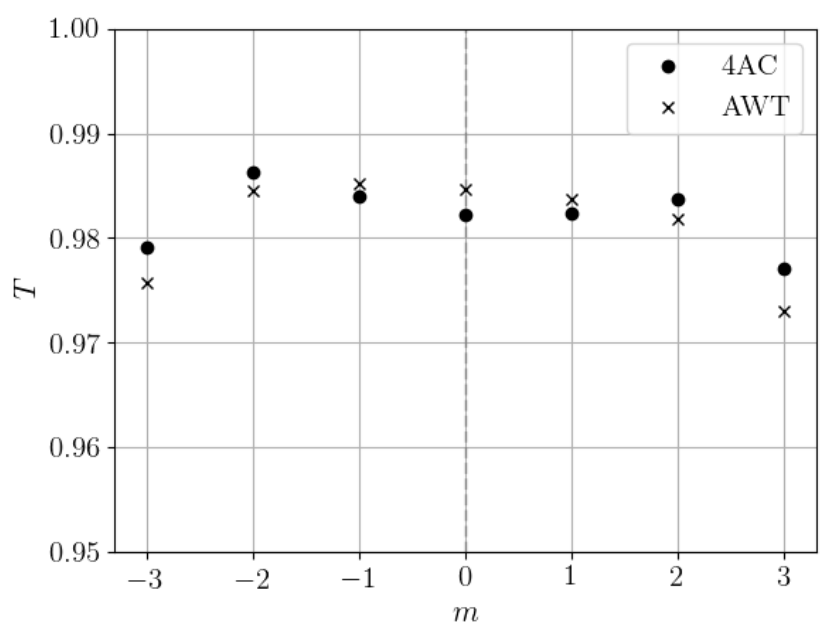

Figure 8 Acoustic transmission coefficient of various simulated circumferential mode orders.

\section{CONCLUSIONS}

In the present numerical study, the proposed scaling approach based on six dimensionless parameters for aeroacoustic similarity was applied to a stator blade row in two test rigs with different shroud radii. By adjusting the aerodynamic and acoustic operating point, the parameters could be matched between the existing compressor blade row and the scaled blade row.

The current CFD results show a good overall agreement of the characteristic parameters up- and downstream of both blades The radial distribution of the Mach and Reynolds number could be matched fairly well between both blades in steady and unsteady simulations. The dimensionless pressure coefficient distribution along the blades' axial chord indicates similar blade loadings and thus, an aerodynamic similarity of the operation points. Furthermore, acoustic simulations of the sound transmission behaviour for a single frequency indicated a similar overall behaviour of the extracted blade row from the multistage axial compressor and the scaled single row blade. The discrepancy of the hub-to-tip ratio between the blade rows predominantly caused the insignificant differences in the obtained acoustic results. As the geometric ratios such as aspect ratio and blade solidity were kept constant while scaling, the minimal deviations in the aerodynamic results were negligible and the hub-to-tip ratio merely impacted the blades' transmission behaviour. In a further aeroacoustic investigation several excitation frequencies are considered to provide a more complete 
comparison. It is expected to see a differing sound transmission behaviour as a result of the hub-to-tip ratio difference when higher radial mode orders are excited.

The presented scaling approach holds the potential to increase the quality of aeroacoustic measurements by scaling selected components of an engine onto geometric dimensions that can be installed in acoustically optimized test rigs. In combination with a flexible air supply system, the reduced operation points can be adjusted and the experimental results can be re-scaled into engine-like conditions.

\section{NOMENCLATURE}

\section{Acronyms}

4AC four-stage high-speed axial compressor

AWT Aeroacoustic Wind Tunnel

CAA computational aeroacoustics

CAD computer-aided design

CFD computational fluid dynamics

GCI grid convergence index (Roache, 1994)

RANS Reynolds-Averaged Navier-Stokes

TFD Institute of Turbomachinery and Fluid Dynamics

URANS Unsteady-Reynolds-Averaged Navier-Stokes

\section{Characteristic Numbers}

$\begin{array}{ll}\sigma_{s} & \text { blade solidity } \\ \mathrm{He} & \text { Helmholtz number } \\ \mathrm{Ma} & \text { Mach number } \\ \mathrm{Re} & \text { Reynolds number } \\ \mathrm{Sr} & \text { Strouhal number }\end{array}$

\section{Greek Symbols}

$\begin{array}{ll}\alpha_{i n c}^{ \pm} & \text {incident wave angle } \\ \alpha_{s} & \text { staggering angle } \\ \eta & \text { dynamic viscosity } \\ \omega & \text { angular frequency } \\ \rho & \text { density } \\ \sigma & \text { hub-to-tip ratio } \\ \tau_{\omega} & \text { wall shear stress }\end{array}$

$\varphi \quad$ circumferential direction/component

\section{Latin Symbols}

$\bar{p} \quad$ complex amplitude of the propagating sound wave

$\hat{p} \quad$ sound pressure

$a_{0} \quad$ speed of sound

$A_{m n}^{ \pm} \quad$ complex amplitudes of the sound pressure

$c \quad$ blade chord length

$c_{p} \quad$ pressure coefficient $\left(c_{p}=\frac{p-p_{\infty}}{\frac{1}{2} \rho_{\infty} v_{\infty}^{2}}\right)$

$c_{\text {rel }} \quad$ relative blade chord length $\left(c_{r e l}=\frac{z-z_{\min }}{z_{\max }-z_{\min }}\right)$

$d \quad$ observed order of accuracy

e error

$f \quad$ frequency

$F_{S} \quad$ factor of safety (grid convergence study)

$F_{y} \quad$ normal force

$F_{z} \quad$ tangential force

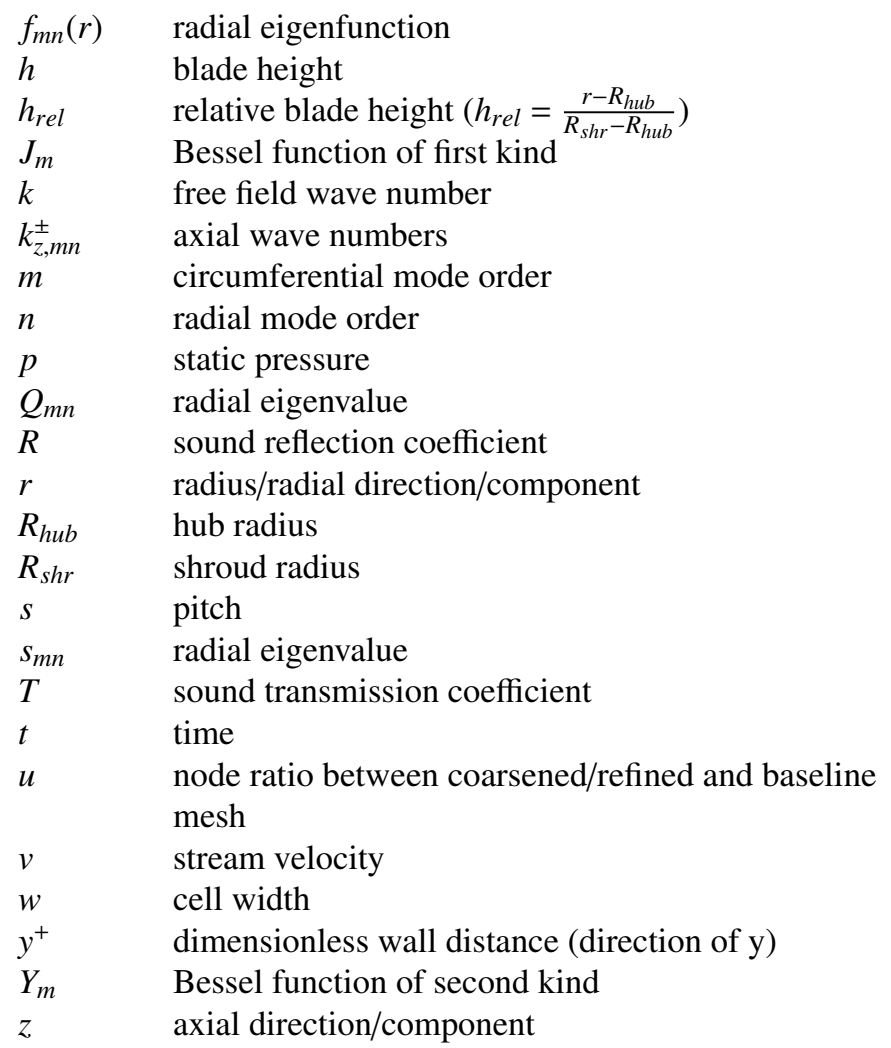

\section{Subscripts}

$\infty \quad$ in the freestream (i.e. remote from any disturbance)

$\varphi \quad$ circumferential

a relative

ext extrapolated

in inlet

inc incoming

out outlet

$r \quad$ reflected

$t \quad$ transmitted

$z \quad$ axial

\section{ACKNOWLEDGMENTS}

The authors would like to thank Compute Canada and the Leibniz Universität Hannover IT Services (LUIS) for providing computational resources. The authors would further like to acknowledge the MSC Software Corporation for providing their software ACTRAN.

\section{REFERENCES}

[1] Hellmich, B. and Seume, J. R. "Causes of Acoustic Resonance in a High-Speed Axial Compressor". In: Journal of Turbomachinery 130 (July 2008).

[2] Hurfar, C. M., Bartelt, M., and Seume, J. R. "A Scaling Method for Modal Sound Propagation in Annular Ducts". In: Proceedings of ASME Turbo Expo 2015: Turbine 
Technical Conference and Exposition. GT2015-42839. Montréal, Canada, 2015.

[3] Smith, S. N. "Discrete Frequency Sound Generation in Axial Flow Turbomachines". In: Reports and Memoranda No. 3709 (Mar. 1972).

[4] Hellmich, B. "Acoustic Resonance In A High-Speed Axial Compressor”. Ph.D. Thesis. Germany: Leibniz Universität Hannover, 2008.

[5] Buckingham, E. "On Physically Similar Systems; Illustrations of the Use of Dimensional Equations". In: Phys. Rev. 4 (June 1914), pp. 345-376.

[6] Behn, M., Tapken, U., Puttkammer, P., Hagmeijer, R., and Thouault, N. "Comparative study of different analytical approaches for modelling the transmission of sound waves through turbomachinery stators". In: Summary of the papers for the 22nd Aeroacoustics Conference (2016).

[7] Weckmüller, C., Guérin, S., and Ashcroft, G. "CFDCAA Coupling Applied to DLR UHBR-Fan: Comparison to Experimental Data". In: Summary of the papers for the 30th AIAA Aeroacoustics Conference (2009).

[8] Antwerpen, B. V., Leneveu, R., and Caro, S. "New advances in the use of Actran/TM for nacelle simulations". In: Proceedings of the 14th AIAA/CEAS Aeroacoustics Conference (May 2008).

[9] Roache, P. J. "Perspective: A Method for Uniform Reporting of Grid Refinement Studies". In: Journal of Fluids Engineering 116.3 (Sept. 1994), pp. 405-413.

[10] Moreau, S., Henner, M., and Neal, D. "3D Rotor-Stator Interaction in Automotive Engine Cooling Fan Systems". In: Proceedings of ETC 2005 (Mar. 2005).

[11] Committee, A. V. Standard for Verification and Validation in Computational Fluid Dynamics and Heat Transfer. The American Society of Mechanical Engineers, 2009. Chap. 2, pp. 13-14.

\section{APPENDIX}

Conversion of the Strouhal number $S r$ to introduce the hub-to-tip ratio $\sigma$ as a dimensionless parameter

Using the dimensionless parameters derived in the dimensional analysis, the following relation is obtained:

$$
\begin{aligned}
S r & =\frac{f \cdot c}{v} \\
& =f \cdot \frac{1}{a_{0} \cdot M a} \cdot \frac{R_{s h r}-R_{h u b}}{\frac{h}{c}} \\
& =\frac{f \cdot R_{s h r}}{a_{0}} \cdot \frac{1}{M a} \cdot \frac{1}{\frac{h}{c}} \cdot \frac{R_{s h r}-R_{h u b}}{R_{s h r}} \\
& =\frac{H e}{2 \pi} \cdot \frac{1}{M a} \cdot \frac{1}{\frac{h}{c}} \cdot(1-\sigma)
\end{aligned}
$$

